\title{
Social Support, Social and Temporal Comparisons Protect Well-Being and Health between 45 and 70 Years Old in Four Urban Communities
}

\author{
Ingrid Gilles ${ }^{1}$, Alain Clemence ${ }^{1^{*}}$, Nelly Courvoisier ${ }^{1}$ and Sylvie Sanchez ${ }^{2}$
}

${ }^{1}$ Psychology Institute, University of Lausanne, Lausanne, Switzerland

${ }^{2}$ Sensory and Consumer Science Group, Nestle Research Center, Lausanne, Switzerland

"Corresponding author: Clemence A, Psychology Institute, University of Lausanne, Batiment Geopolis CH-1015 Lausanne, Switzerland, Tel: +021 692 3240 , Fax: +41 21692 315; E-mail: alain.clemence@unil.ch

Received date: March 06, 2015; Accepted date: April 13, 2015; Published date: May 08, 2015

Copyright: @ 2015 Gilles I, et al. This is an open-access article distributed under the terms of the Creative Commons Attribution License, which permits unrestricted use, distribution, and reproduction in any medium, provided the original author and source are credited.

\begin{abstract}
This study examined the impact of social support and of temporal and social comparisons on well-being and selfreported health in four capital cities: Paris, Berlin, Moscow and Beijing. Based on the lifespan control theory, an integrative model investigating the influence of these coping strategies, especially on the psychological regulation of health losses, was tested on 1141 respondents aged 45 to 70 years by using structural equation modelling with multigroup comparisons. Results indicated a good fit of the model to participants' responses. In all contexts, physical weaknesses favoured the use of social and temporal comparison strategies rather than social support. Moreover, across the cities, coping strategies were oriented more toward protecting self-evaluation of health than toward enhancement of well-being. Social comparison decreased the impact of physical weaknesses on health evaluation and on well-being in the four cities, but to a lesser extent in China. Results are discussed regarding the normative cross-cultural aspects that intervene during ageing in the four urban contexts.
\end{abstract}

Keywords: Aging; Well-being; Perceived health; Coping strategies; Social comparison; Temporal comparison; Social support; Crosscultural comparison

\section{Introduction}

Ageing is characterised by increasing losses of physical and cognitive abilities, activities and relationships [1]. After relative stability during adulthood, the losses become less balanced by gains and contribute to an individual's decreasing sense of control over the external environment. However, a number of studies have demonstrated that people adapt to this change by activating coping mechanisms and mobilising resources to maintain a high satisfaction with life [2-4]. For example, the theory of the lifespan control elaborated by Heckhausen and Schulz [5-7] offers a strong framework to analyse the optimal processes used to regulate ageing, in particular the protection of health as a superordinate goal. From this perspective, individuals use compensatory strategies of control targeting their environment (primary control) and the self (secondary control). In the present study, we examine two specific compensatory control strategies, namely social support and social/temporal comparison from midlife to the beginning of old age, used to maintain positive perception of health and well-being. Although the effectiveness of these compensatory controls is well established for older people, less research has tested it from midlife to the beginning of old age [8]. Thus, the first objective of the present study was to study the effectiveness of social support and social/temporal comparison as compensatory controls from midlife to the beginning of old age. Moreover, the universality of the lifespan theory of control claimed by Heckhausen and Schulz [9,10] was challenged by Gould [11], and few comparatives studies have been conducted to examine its effectiveness. As a consequence, another objective was to test these control strategies in four different sociocultural contexts.

\section{Social support and social/temporal comparisons}

People have different resources at their disposal to face disturbing events and more generally the inevitable biological degradation that affects health. For instance, socio-economic resources are known to offer important protection [12,13], and social support is also an important buffer against increasing stress caused by losses and negative events in later life. People benefit not only from the help of a social network, including family and relatives, but also from membership in social groups and the recognition that these groups offer their members [14,15]. Integration in a social network is a primary control strategy to stimulate activities and compensate possible functional incapacities in everyday life [16]. Although the processes linking social support to the status and perception of health need to be more rigorously characterised, an abundant literature has demonstrated the positive regulation on well-being of social ties and social support $[17,18]$.

Social and/or temporal comparisons are typical compensatory secondary controls [19]. As people get older, disease and death become more salient in their immediate environment. Overall, this salience of negative events may induce a feeling of threat to one's own health. As stated in the theory of cognitive adaptation [20-22]. Overestimation of their health status helps people to cope effectively with physical weakness. Instead of attempting to control irreversible modifications, they adopt a strategy of coping that consists in transforming representations of the self and of their environment in an optimistic way. Such secondary control offers the possibility of integrating disturbing events by considering them as normal changes in the lifespan [6,23]. The self-enhancement of health and evaluations of well-being are based on a downward comparison with peers of the same age [24]. Because the opportunity to select a real and symbolic target of comparison who has worse health status than the selfincreases with age, the influence of social comparison exerts a stronger 
compensatory function when people get older [25]. The beneficial function of a temporal comparison was shown in preliminary work on its negative impact on health evaluation in elderly people [26]. However, recent studies demonstrate that temporal comparison favours the preservation of a positive self-evaluation of health and well-being by moderating the level of expectations in ageing $[9,19]$.

The relative impact of these two coping strategies on evaluations of health and well-being has been largely documented in Western populations, but scarcely in other cultural contexts, such as in Russia and China [27]. The aim of the present study was to document this aspect by examining the buffering impact of social support and of both kinds of comparison on well-being and self-reported health in four cultural urban contexts (Paris, Berlin, Moscow and Beijing).

\section{Socio-cultural differences}

The experience and representations of ageing are shaped by different cultures, as shown, for example, by traditional studies that compared Eastern and Western countries [11,28,29]. Ageing appears to be more positively viewed in China and Russia than in France and other Western countries [30]. Compared with Western countries, in China and Russia, this view is partially anchored in the sociocultural tradition, but also in the important societal changes that occurred there in the last several decades. Life expectancy increased considerably in China, especially in urban areas such as Beijing, where the mean age, over 80 years, is similar to that in Western countries [31]. In Russia, in contrast, life expectancy dramatically decreased, especially for men, after the collapse of the Soviet Union: in 2011, life expectancy was 63 years for men and 75 years for women [32,33]. The statutory retirement age in these countries nevertheless occurs at a similar age range. It was fixed in 2009 at 65 years old in Germany, 60 years old in China and France, and 60 years old for men and 55 years old for women in Russia [34]. China and Russia have less institutional support at their disposal to care for elderly people than do Western countries [32,35]. In addition, during the same period, the family network narrowed under the "only child" policy in China, an evolution that can be compared with the dramatic reduction in the birth rate in Russia and most European countries. This change, however, did not have the same meaning in Beijing as it did in the other three cities. In Beijing, older adults are married, with few living alone. Furthermore, "Chinese culture has a unique set of norms that prioritize family over the individual" [36]. In China, social support is linked to intergenerational relationships, which have long been influenced by a norm of reciprocity, whereas in Western countries it is linked to older adults' empowerment and is a sign of independence [35]. This implies that, to maintain a high level of well-being and health self-evaluation, the support of the social network (family and institutional) is more relevant in France and Germany than it is in China. In Russia, family support appears to be more related to the socio-economic situation than it does in China. In Russia, compensation for the deterioration of institutional support caused by socio-economic problems better explains the generations living together than tradition do $[32,37]$.

There is relative consensus, however, about lifespan characterised by the increasing paradox during ageing of age-related declines in physical health and stability in well-being $[38,39]$. This suggests that compensatory control strategies such as social comparisons and, to a lesser extent, temporal comparisons are more universal strategies than social support is. Indeed, if the integration in social networks, as a primary control, strongly depends on societal norms, as evoked earlier, the self-enhancement that results from social and temporal comparisons appears to be anchored in a cross-culturally shared representation of normal lifespan, including the degradation of physical capacities and the motivation to maintain a positive view of proper health $[10,38]$. Consequently, these cognitive processes should be more salient and universal than social support in cross-cultural comparisons to reduce the impact of health deterioration on wellbeing and health self-evaluation.

\section{The present study}

From these arguments, we suggest a general model to test the decline in physical functioning in association with the relative stability of well-being and health self-evaluation during ageing (Figure 1). On the one hand, weakening of health should increase the recourse to social support, which should, in turn, positively influence well-being and the perception of health. On the other hand, temporal and social comparisons should enhance well-being and health perception, and should partially compensate for the negative impact of weakening health. Indeed, even if people adjust temporal and social comparisons to their physical state, they should also overestimate their health when comparing it with that of others and, to a lesser extent, to the past. We expect a stronger impact of social support in Paris, Berlin and Moscow than in Beijing, the latter of which is based more on norms regarding family support, as well as similar temporal and social comparison effects among the four populations. This line of reasoning assumes age-related stability not only of well-being and self-evaluated health, but also of temporal and social comparisons, to support the assumption of overestimating self-evaluation in each country.

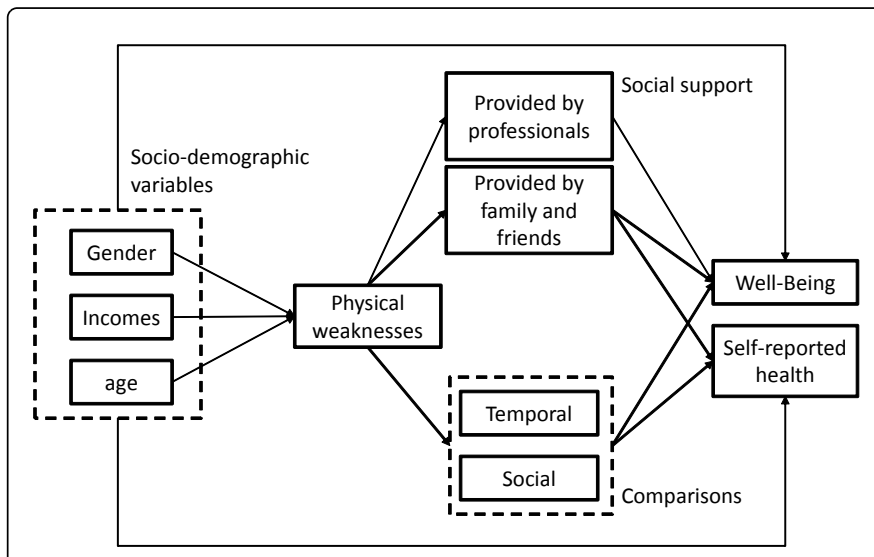

Figure 1: Hypothesised model: buffering effect of social support and comparisons of well-being and self-reported health.

\section{Method}

\section{Participants and procedure}

Questionnaires were completed by inhabitants of the capital cities in four countries: France ( $N=353)$, Germany $(\mathrm{N}=349)$, Russia $(\mathrm{N}=362)$ and China $(\mathrm{N}=350)$. Marketing agencies recruited respondents by using quota sampling based on gender, three age categories (45-55; $56-65 ; 66-75)$ and three classes of income adjusted to each country's standards (low, medium and high). Ads were published in local newspapers, and potential participants were questioned to confirm that they fit the quotas. They were then invited, by groups of about ten, 
Page 3 of 9

to respond to the survey in the agency offices. An agency employee, blind to the study purpose, was present during each session to answer questions and to ensure that each respondent filled out the questionnaire individually. Respondents received payment for their participation corresponding to $1 \mathrm{hr}$ of work in the country's capital. Recruitment problems led to the Chinese sample consisting only of respondents who were less than 70 years. We thus eliminated participants over 70 years in the three remaining samples, adjusting the Chinese sample by randomly discarding a similar number of younger respondents $(\mathrm{N}=273)$. Our final sample included 1141 respondents: 283 in each of France and Germany, 285 in Russia and 290 in China.

\section{Measures}

Respondents completed a paper-and-pencil questionnaire, which was translated into the language of each country. To deal with translation issues and potential misunderstanding of concepts because of social and cultural contexts, we used complementary interviews conducted by one of the authors to control for concept equivalence. Means and standard deviations of the measures are presented in Table 1 for each country.

\section{Social support}

We proposed a composite measure of perceived social support that included three indicators [40]. Respondents estimated (1) the size of their close social network by naming relatives, friends and professionals who provided support; (2) the frequency of support by indicating the number of times they had met each person during the last year, on a 5 -point Likert scale ( $1=$ less than once a month, $5=$ every day); and (3) the importance attributed to each relationship, on a 3point Likert scale $(1=$ not essential at all, to $3=$ very essential). Mean scores were computed for the frequency and the importance of the support. For each indicator, we differentiated the support provided by friends or family from the support provided by professionals.

\section{Coping strategies}

Social and temporal comparisons were assessed with two single items [41]. The respondents evaluated their health, on a 5-point scale ( $1=$ much poorer, $5=$ much better), compared with their peers' health (social comparison) and their own health 1 year earlier (temporal comparison).

\section{Physical weaknesses}

This measure provided a global estimate of recently experienced chronic health issues. A list of 13 chronic health issues (e.g. allergy, asthma, diabetes, heart attack) adapted from the Mini European Health Module [42] was given to the respondents, who indicated whether they had experienced each issue several times (coded 1), once (coded 0.5 ) or never in the last few weeks (coded 0). A sum score $(0=$ no physical weaknesses experienced, $13=$ all physical weaknesses experienced several times in the last few weeks) was then computed (France: alpha $=0.72$; Germany: alpha $=0.64$; China: alpha $=0.72$; Russia $=0.76$ ).

\section{Self-reported health}

This was assessed by a single item "In general, would you say that your health is?" on a 5 -point scale ( $1=$ really bad, $5=$ really good) [42].

\section{Well-being}

We used the eight-item version of the Self-Assessing Depression Scale [43] to measure well-being. This scale includes positive (e.g. I enjoy what I do, I feel confident, I trust in the future) as negative (e.g. I feel anxious, I feel sad) affective states, and has the advantage to be directly applicable to the present life of individuals. So, the instrument is not restricted to a measure of depressive symptoms, and has also been used to measure a general state of well-being in the daily life [44]. A mean score (1=low well-being, 5=high well-being) was computed (France: alpha $=0.82$; Germany: alpha $=0.72$; China: alpha $=0.60$; Russia $=0.61)$.

\section{Socio-demographic variables}

Respondents were asked to indicate age, gender, marital status, number of children and other information concerning education level and employment. Respondents were assigned one of three income classes.

\section{Analyses}

Multigroup structural equation modelling with maximum likelihood estimations (using AMOS 19 software) was conducted following the Byrne procedure for multigroup invariance testing [45]. Our hypothesised model was tested and re-specified for each group separately until a baseline of well-fitting models was obtained. These baseline models were then combined in a configural multigroup model, which was tested on the four groups simultaneously without constraints. To test variations or invariances between groups, we compared the non-constrained configural model (M1) to different constrained models: first, to a model in which factor loadings and error covariance were constrained to equality across countries (M2); second, to a model in which factor loadings, error covariance and factor variance were constrained to equality across countries (M3); and third, to the independence model in which all paths were constrained to zero (M4). Differences between countries could be claimed when model comparisons favoured the non-constrained configural model (M1).

Path models were chosen over analyses with latent variables to ensure stability of the variables in the model and because the model included many single-item measures [46]. The goodness-of-fit of a model was determined from the following indices: relative chi-square $\left(\chi^{2} / \mathrm{df}\right.$; should be $\leq 2.0$ [47]), root mean square error of approximation (RMSEA; should be $\leq 0.06$ [48]), comparative fit index (CFI; should be $\geq 0.95$ [49]) and standardised root mean square residual (SRMR; should be $\leq 0.08$ [47]). We checked for the linearity and normality (outcome and measures). Appropriate transformations were performed for skewed distribution. Missing cases were marginal (less than 1 per cent per variable) and were thus imputed using a regression method. Finally, linear regressions confirmed the non-colinearity between the variables in the models.

\section{Results}

\section{Respondent's characteristics}

Table 1 presents characteristics of the respondents. We observed that those from France and Germany had close patterns in regard to marital status, number of children and last occupation held. More particularly, for both countries, about 25 per cent of the respondents 
Citation: Ingrid Gilles, Alain Clemence, Nelly Courvoisier and Sylvie Sanchez (2015) Social Support, Social and Temporal Comparisons Protect Well-Being and Health between 45 and 70 Years Old in Four Urban Communities. Aging Sci 3: 133. doi:10.4172/2329-8847.1000133

Page 4 of 9

were married, single or divorced; about 50 per cent had between one and two children; and between 20 and 30 per cent were managers. The Chinese sample was characterised by a strong majority of married people having one child. These data do not correspond to the current evolution of these two indicators in China because the one-child policy is now questioned owing to its role in the ageing of the Chinese population [50] and because divorce has noticeably increased since the eighties [51].

\begin{tabular}{|c|c|c|c|c|}
\hline & Paris \% & Berlin \% & Beijing \% & Moscow \% \\
\hline \multicolumn{5}{|l|}{ Gender } \\
\hline Men & 50.9 & 49.8 & 51.0 & 53.7 \\
\hline Women & 49.1 & 50.1 & 49.0 & 46.3 \\
\hline \multicolumn{5}{|l|}{ Age } \\
\hline $45-55$ & 42.4 & 41.3 & 44.8 & 42.1 \\
\hline $56-65$ & 40.6 & 42.0 & 47.6 & 42.5 \\
\hline $66-70$ & 17.0 & 16.6 & 7.6 & 15.4 \\
\hline Mean (SD) & $57.42(7.34)$ & $56.82(7.50)$ & $56.11(6.96)$ & $56.67(7.20)$ \\
\hline \multicolumn{5}{|l|}{ Marital status } \\
\hline Single & 25.8 & 29.3 & 0.7 & 17.2 \\
\hline Partners & 16.3 & 17.0 & 0.7 & 48.4 \\
\hline Married & 28.6 & 29.7 & 95.9 & 22.8 \\
\hline Divorced & 25.1 & 18.0 & 1.7 & 11.6 \\
\hline Widower & 4.2 & 6.0 & 1.0 & 0.0 \\
\hline \multicolumn{5}{|l|}{ Number of children } \\
\hline 0 & 27.9 & 33.6 & 2.8 & 14.4 \\
\hline 1 & 25.4 & 28.6 & 76.6 & 34.7 \\
\hline 2 & 30.7 & 27.9 & 19.7 & 40.4 \\
\hline 3 & 11.7 & 8.1 & 0.7 & 9.1 \\
\hline 4 and more & 4.3 & 1.8 & 0.3 & 1.4 \\
\hline \multicolumn{5}{|l|}{ Studies $^{a}$} \\
\hline Yes & 92.6 & 97.9 & 98.6 & 89.8 \\
\hline No & 7.4 & 2.1 & 1.4 & 10.2 \\
\hline \multicolumn{5}{|l|}{ Last occupation } \\
\hline Farmer & 0.0 & 0.4 & 0.7 & 0.4 \\
\hline Independent occupation & 26.8 & 22.6 & 30.0 & 14.0 \\
\hline Manager & 28.6 & 18.7 & 0.0 & 1.4 \\
\hline Employee & 42.4 & 51.9 & 37.9 & 63.5 \\
\hline Worker & 1.1 & 5.3 & 31.0 & 18.2 \\
\hline Without & 1.1 & 1.1 & 0.3 & 2.5 \\
\hline
\end{tabular}

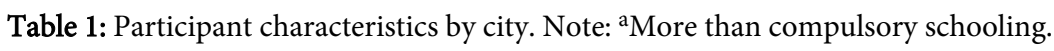

These data are consistent, however, with our specific population because the respondents got married and had their child during the time of the one-child policy [52] and when the negative perception of divorce was prominent. Concerning occupations, the Chinese sample did not have any managers, but was mainly composed of employees, workers and people with independent occupations. The Russian 
Citation: Ingrid Gilles, Alain Clemence, Nelly Courvoisier and Sylvie Sanchez (2015) Social Support, Social and Temporal Comparisons Protect Well-Being and Health between 45 and 70 Years Old in Four Urban Communities. Aging Sci 3: 133. doi:10.4172/2329-8847.1000133

Page 5 of 9

sample was characterised by almost 50 per cent of people cohabiting outside of marriage and living as a couple and 86 per cent of respondents having between one and two children. Russian respondents were predominantly employees or workers.

We observed that, for all samples, more than 90 per cent of respondents said they had studied beyond compulsory schooling, which is higher than the official rate in each country (about 80 per cent according to the national census).

\section{Inter-and intra-country differences}

A multivariate analysis of variance was conducted on the different measures, with countries, income classes and sex as between-subject factors and age as covariate. The main statistically significant effects at $\mathrm{p}<0.001$ are shown below. No interaction effects were significant.

\section{Countries}

As shown in Table 2, there are clear differences between countries for weaknesses and self-reported health, and for well-being, all Fs (3, 1141) $>13.52 ; \mathrm{p}<0.001$. Chinese and, to a lesser extent, German respondents reported fewer physical weaknesses than other groups. Russian respondents indicated a lower level of well-being and subjective health than French respondents and, to a greater extent, than German and Chinese respondents. In Beijing and Moscow, participants cited a smaller social network, but more frequent contacts than did those living in Paris and Berlin, both Fs $(3,1141)>86.09$; $\mathrm{p}<0.001$. The social network was more important for Russian participants than for French, German and Chinese participants, $\mathrm{F}$ $(3,1141)=60.01, p<0.001$. No differences between countries appeared for both dimensions of health comparison.

\begin{tabular}{|l|l|l|l|l|l|l|l|l|}
\hline & \multicolumn{2}{|l}{ Paris } & \multicolumn{2}{l}{ Berlin } & \multicolumn{2}{l|}{ Beijing } & \multicolumn{2}{l|}{ Moscow } \\
\cline { 2 - 9 } & M & SD & M & SD & M & SD & M & SD \\
\hline Physical weakness & 3.34 & 1.83 & 2.43 & 1.5 & 1.95 & 1.38 & 3.3 & 1.81 \\
\hline Self-reported health & 3.56 & 0.8 & 3.75 & 0.75 & 3.89 & 0.67 & 3.3 & 0.61 \\
\hline Well-being & 3.81 & 0.77 & 4.01 & 0.67 & 3.95 & 0.59 & 3.69 & 0.69 \\
\hline Social comparison & 3.64 & 0.89 & 3.73 & 3.73 & 3.64 & 0.84 & 3.57 & 0.93 \\
\hline Temporal comparison & 2.92 & 0.7 & 2.89 & 2.89 & 3.05 & 0.76 & 3.02 & 0.79 \\
\hline Size of close network & 6.8 & 3.24 & 8.58 & 3.08 & 4.33 & 2.49 & 3.38 & 2.4 \\
\hline Size of professional network & 3.69 & 2.22 & 3.33 & 2.16 & 1.53 & 1.46 & 1.11 & 1.09 \\
\hline Frequency of close contacts & 3.26 & 0.92 & 3.11 & 0.72 & 3.77 & 0.8 & 3.87 & 0.92 \\
\hline Frequency of professional contacts & 1.89 & 0.79 & 1.77 & 0.83 & 2.28 & 1.07 & 2.27 & 1.31 \\
\hline Importance of close support & 2.45 & 0.41 & 2.58 & 0.33 & 2.46 & 0.47 & 2.76 \\
\hline Importance of professional support & 2.32 & 0.54 & 2.3 & 0.48 & 1.95 & 0.58 & 2.56 & 0.41 \\
\hline
\end{tabular}

Table 2: Physical weakness, self-reported health, well-being, health comparisons and social support by city. Note: Physical weakness=scale from 0 through 13; self-reported health, well-being, social comparison and temporal=all scales from 1 through 5 ; size of network=number from 0 through 12; frequency of contacts: scale from 1 through 5; importance of support=scale from 1 through 3 .

\section{Incomes}

Well-being and self-reported health increased with higher incomes, Fs $(2,1141)>9.32 ; \mathrm{p}<0.001$. Moreover, the number of people providing social support and positive social comparison increased with higher incomes, Fs $(1,1141)>7.30 ; \mathrm{p}<0.001$.

\section{Gender}

Men reported a better physical state than women, $\mathrm{F}(1,1141)=30.45$; $\mathrm{p}<0.001$.

As expected, social comparison led to an overestimation of health: less than 10 per cent of the participants in each city evaluated their health as being worse than that of their peers, whereas more than 49 per cent judged it to be better. For approximately two thirds of the respondents, health had not changed since the preceding year, and for the remaining one third, it had either increased or decreased (equivalent proportions). As shown in Figure 2, perceived health, wellbeing and health comparison with peers were more optimistically evaluated than physical weaknesses, in particular in Paris and Berlin (Figure 2). The figure also confirms the existence of differences in physical health between countries and suggests an influence of social comparisons in each city. However, only the test of the hypothesised model can answer the question of mediation exerted by social (and temporal) comparison in the self-enhancement process.

\section{Construction of the configural model}

In our hypothesised model (Figure 1), we expected gender, age and incomes to have an indirect impact on well-being and self-reported health, that is, women, older adults and low-income participants to report more physical weaknesses. More physical weaknesses would increase social support (importance, size and frequency) provided by professionals and by family and friends, and would be negatively associated with temporal and social comparisons regarding health. Each aspect of social support and both types of comparison would increase well-being and self-evaluated health. But we also expected a direct effect of the three socio-economic variables on well-being and 
Citation: Ingrid Gilles, Alain Clemence, Nelly Courvoisier and Sylvie Sanchez (2015) Social Support, Social and Temporal Comparisons Protect Well-Being and Health between 45 and 70 Years Old in Four Urban Communities. Aging Sci 3: 133. doi:10.4172/2329-8847.1000133

Page 6 of 9

self-reported health. Initial testing of this model yielded a poor fit to the data for all countries $\left(\chi^{2} / \mathrm{df}>7.96, \mathrm{CFI}=0.87\right.$, SRMR $=0.06$ and RMSEA $=0.08$ [0.07-0.09]). Further testing of the model on each country revealed that physical weaknesses directly decreased wellbeing and self-reported health. Moreover, among the social support variables, only the frequency of support provided by family and friends or by professionals was relevant for the model. Removing the size of the social network and the importance of the support improved the models. Similarly, the models were significantly improved by removing age from the calculations. Indeed, chronological age was not significantly related to any variable in each country.

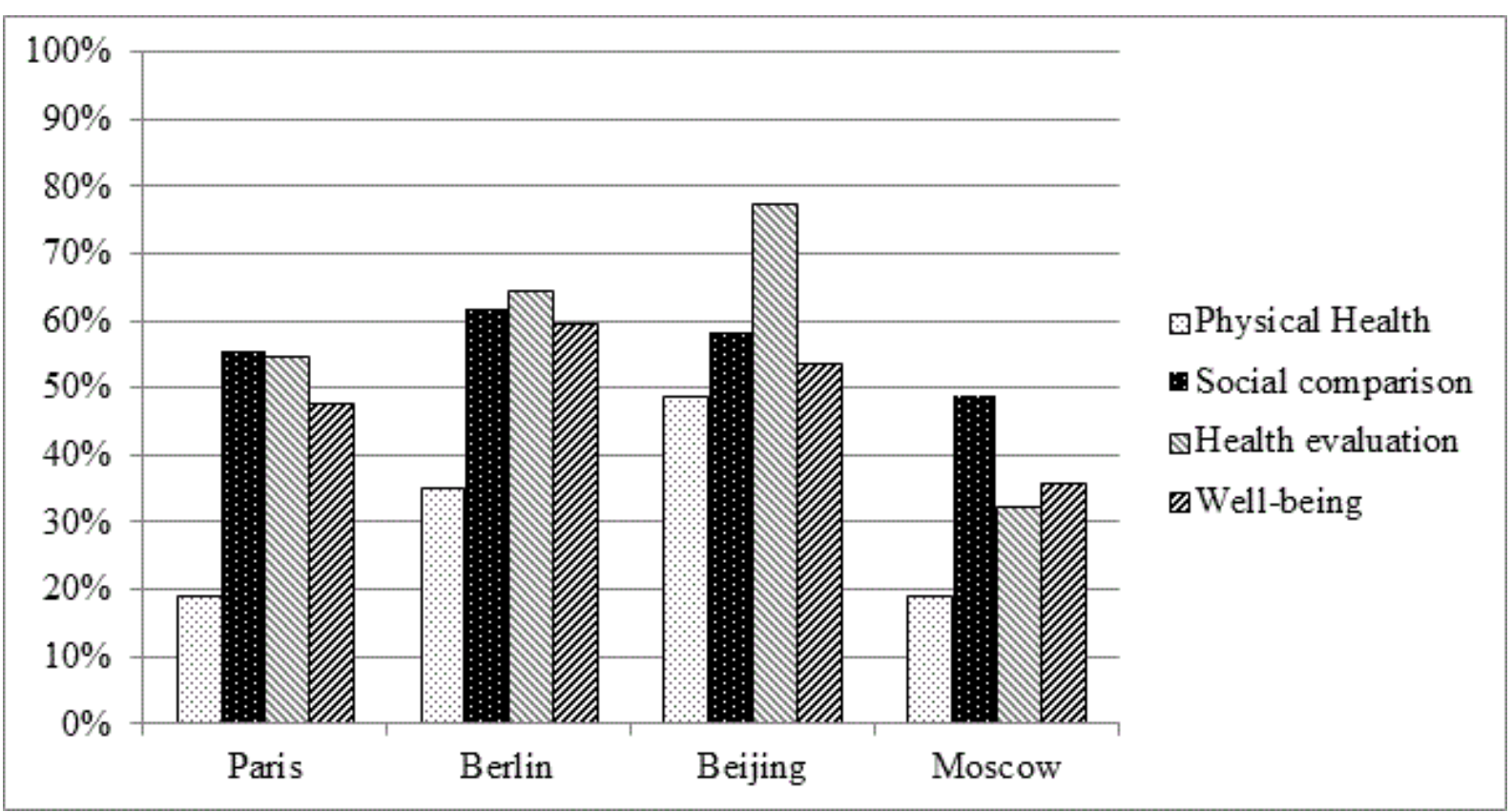

Figure 2: Self-enhancement in social comparison, health evaluation and well-being compared with physical health in four cities. Note: Percentages represent the participants indicating 4 and 5 in social comparison of their health to peers ("somewhat better" and "much better"), health evaluation ("quite good" and "really good") and well-being ("not really" and "not at all apply" about eight depressive and anxiety symptoms). The percentage of the physical weakness clusters at a third of all participants having less than two physical problems.

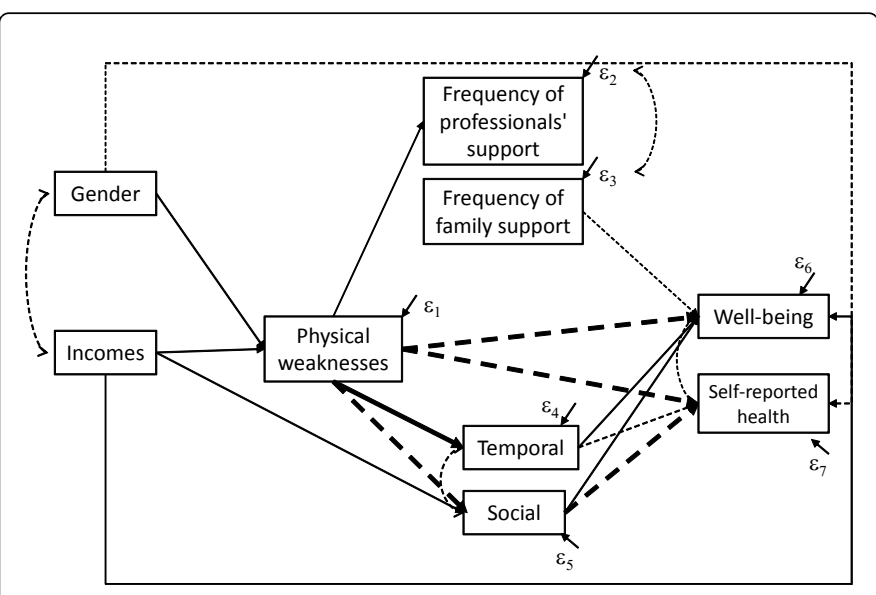

Figure 3: Significant paths in the configural multigroup model. Note: Continuous (double) arrows: equal factor loadings or correlations across countries. Broken (double) arrows: different factor loadings or correlations between countries. Bold continuous or broken arrows: stronger loadings, betas $>0.20$. $\varepsilon 1$ to $\varepsilon$ 8: endogenous variable disturbances.
Finally, incomes had a direct impact on the frequency of the support received from professionals and family or friends and comparison variables. For all countries, this corrected model led to four similar baseline models representing a significant improvement (all samples: $\left.\Delta \chi^{2}(36)>331.5 \mathrm{p}<0.001\right)$ with a good fit (all samples: $\chi^{2} /$ $\mathrm{df}<1.56$, CFI $>0.99$, SRMR $<0.03$, RMSEA $<0.04)$. Baseline models among the four countries were then combined in a unique configural model (Figure 3), allowing us to explore differences between countries.

\section{Test of the configural model: equivalences and differences between countries}

Summary of fit indices and model comparisons (Table 3) indicate that constrained models (M2 to M4) always had poorer goodness-offit statistics than the configural model (M1). We concluded that there were significant differences between factor loadings and covariances in the model because of country differences. To identify these differences, we conducted successive tests of the configural model, constraining one path at a time. Considering the size of the four samples, we present the standardized path coefficients $(\beta)$ by taking into account the power of the effect size: given an $\alpha=0.05$ and $n=280$, a statistically significant coefficient higher than 0.18 (power $>0.90$, one tail test) was qualified as strong, a coefficient between 0.14 and 0.18 as acceptable (power $\approx 0.80$ ) and a coefficient lower than 0.14 as modest. 
Citation: Ingrid Gilles, Alain Clemence, Nelly Courvoisier and Sylvie Sanchez (2015) Social Support, Social and Temporal Comparisons Protect Well-Being and Health between 45 and 70 Years Old in Four Urban Communities. Aging Sci 3: 133. doi:10.4172/2329-8847.1000133

Page 7 of 9

\begin{tabular}{|l|l|l|l|l|l|l|l|}
\hline Model & $\mathbf{X}^{2}$ df & CFI & SRMR & RMSEA (90\% Cl) & Model comparison & $\boldsymbol{\Delta d f}$ & $\Delta \mathbf{x}^{2}$ \\
\hline M1 & 1.08 & 0.99 & 0.02 & $0.01(0.00,0.02)$ & - & & \\
\hline M2 & 2.02 & 0.89 & 0.07 & $0.03(0.02,0.04)$ & M1 - M2 & 84 & $199.89^{* * *}$ \\
\hline M3 & 2.20 & 0.85 & 0.07 & $0.03(0.03,0.04)$ & M1 - M3 & 111 & $280.39^{* * *}$ \\
\hline M4 & 9.06 & 00 & - & $0.08(0.08,0.09)$ & M1 - M4 & 116 & $1269.97^{* * *}$ \\
\hline
\end{tabular}

Table 3: Goodness of fit statistics and model comparison between the configural model and alternative models. Note: ${ }^{* *} \mathrm{p}<0.001 ; \mathrm{M} 1=\mathrm{not}$ constrained configural model; $\mathrm{M} 2=$ constrained model with equal factor loading and error covariance across countries; M3=constrained model with equal factor loading, error covariance and factor variance across countries; M4=independence model; $\chi^{2} / \mathrm{df}=\mathrm{chi}$ square to $\mathrm{df}$ ratio; $\mathrm{CFI}=$ robust version of the Comparative Fit Index; SRMR=standardised root mean square residual; RMSEA= robust version of the root mean square error of approximation; $\mathrm{CI}=$ confidence interval; $\Delta \mathrm{df}=$ difference of degree of freedom between two models to be compared; $\Delta \chi^{2}$ $=$ difference between chi squares of two models to be compared.

\section{Equivalence between countries}

Concerning socio-demographic factors, we observed a significant effect of gender on physical weaknesses: women reported more physical weaknesses in all countries, $\beta[0.15,0.14] \mathrm{ps}<0.001$. Incomes had a modest but systematic positive impact on physical weaknesses (fewer physical weaknesses; $\beta[-0.07,-0.08]$ ps $<0.05$ ), social comparison $(\beta[0.11,0.09] \mathrm{ps}<0.001)$ and well-being $(\beta[0.11,0.10]$ ps<0.001).

In the second step of the model, physical weaknesses were strongly negatively associated with temporal comparisons $(\beta[-0.22,-0.24]$ ps $<0.001)$. They were also, quite logically, positively linked with the frequency of support provided by professionals, but this link remained modest $(\beta[0.07,0.09]$ ps $<0.01)$.

In the final step, social and temporal comparisons had positive modest effects on well-being $(\beta[0.11,0.10] \mathrm{ps}<0.001$ and $\beta[0.07,0.05]$ ps $<0.05$, respectively), and social comparison strongly increased selfevaluation of health across the four countries, $\beta[0.43,0.34] \mathrm{ps}<0.001$.

\section{Differences between countries}

Variations between countries (cf. Table 4) concerned mainly the impact of physical weaknesses on other variables. Results showed a clear contrast between how physical weaknesses affected social comparison, well-being and self-reported health among the Chinese and the other respondents. Among respondents with physical weaknesses, Chinese participants made fewer negative social comparisons and reported less of a decrease in well-being and selfreported health than did Russian, German or French participants.

We also observed that the effect of social and temporal comparisons on self-reported health varied across countries. Temporal comparison increased self-reported health for all respondents except for those from China, whereas social comparison strongly increased self-reported health among all respondents, but to a lesser extent for those from Russia.

Finally, the frequency of the support provided by family and friends did not influence self-reported health, but it significantly increased the well-being of French and German respondents. It had no impact on the well-being of Chinese and Russian respondents. The frequency of the support provided by professionals had no impact on our two outcomes.
Globally, the configural model explained a reasonable proportion of well-being and self-reported health variances (cf. Table 4). However, it better explained the well-being variance in French, German and Russian samples than in the Chinese sample. Similarly, the configural model better explained self-reported health variance in French and German samples than in Chinese and Russian samples.

\begin{tabular}{|c|c|c|c|c|c|c|}
\hline \multirow[b]{2}{*}{$\begin{array}{l}\text { Depende } \\
\text { nt } \\
\text { variables }\end{array}$} & \multicolumn{5}{|c|}{ Influencing variables } & \multirow[b]{2}{*}{$\mathbf{R} 2$} \\
\hline & Gender & $\begin{array}{l}\text { Physica } \\
\text { I } \\
\text { weakne } \\
\text { ss }\end{array}$ & $\begin{array}{l}\text { Frequen } \\
\text { cy of } \\
\text { family } \\
\text { support }\end{array}$ & $\begin{array}{l}\text { Temporal } \\
\text { comparis } \\
\text { on }\end{array}$ & $\begin{array}{l}\text { Social } \\
\text { comparis } \\
\text { on }\end{array}$ & \\
\hline $\begin{array}{l}\text { Social } \\
\text { compariso } \\
\mathrm{n} \\
\text { France } \\
\text { Germany } \\
\text { China } \\
\text { Russia }\end{array}$ & & $\begin{array}{l}-0.32^{* * *} \\
-0.37^{\star * *} \\
-0.16^{* *} \\
-0.32^{\star * *}\end{array}$ & & & & \\
\hline $\begin{array}{l}\text { Well-being } \\
\text { France } \\
\text { Germany } \\
\text { China } \\
\text { Russia }\end{array}$ & & $\begin{array}{l}-0.31^{* * *} \\
-0.37^{\star * *} \\
-0.12^{*} \\
-0.39^{\star * *}\end{array}$ & $\begin{array}{l}0.21^{* * *} \\
0.11^{*} \\
-0.02 \\
0.05\end{array}$ & & & $\begin{array}{l}0.29 \\
0.22 \\
0.09 \\
0.21\end{array}$ \\
\hline $\begin{array}{l}\text { Self- } \\
\text { reported } \\
\text { health } \\
\text { France } \\
\text { Germany } \\
\text { China } \\
\text { Russia }\end{array}$ & $\begin{array}{l}0.05 \\
0.04 \\
-0.09 \\
-0.13^{*}\end{array}$ & $\begin{array}{l}-0.31^{\star \star *} \\
-0.26^{\star * *} \\
-0.15^{\star *} \\
-0.25^{\star * *}\end{array}$ & & $\begin{array}{l}0.15^{\star *} \\
0.17^{\star \star *} \\
0.02 \\
0.12^{*}\end{array}$ & $\begin{array}{l}0.39^{\star \star *} \\
0.47^{\star \star *} \\
0.38^{\star \star *} \\
0.27^{\star * *}\end{array}$ & $\begin{array}{l}0.44 \\
0.51 \\
0.23 \\
0.27\end{array}$ \\
\hline
\end{tabular}

Table 4: Configural multigroup model: standardised path coefficients varying between countries and R-square outcomes. Note: ${ }^{*} \mathrm{p}<0.05$; ${ }^{* *} \mathrm{p}<0.01 ;{ }^{* * *} \mathrm{p}<0.001$. On the basis of an $\alpha=0.05$ and $n=280, \beta \geq 0.18$ correspond to a strong effect size (power $>0.90$, one tail ), 0.180 .14 correspond to a moderate effect size (power $\approx 0.80$ ), $\beta \leq 0.14$ correspond to a modest effect size (power $<0.80$ ). 
Page 8 of 9

\section{Discussion}

Research on ageing highlights the role of social support and temporal and social comparisons in self-reported health and wellbeing. These compensatory mechanisms have rarely been conjointly studied from a cultural comparison perspective [27]. Our results extend the findings on the compensatory effect of social and temporal comparisons on self-rated health and well-being in two directions: by stressing its effectiveness in a cross-cultural context and by comparing people from the end of midlife to the beginning of old age. They also highlighted variations between countries regarding the impact of physical weakness and social support.

\section{Equivalences across countries}

The most basic similar process across countries was an indirect impact of physical weaknesses on both outcomes through temporal and social comparisons of health. The strong and moderate positive effects of social comparison on self-rated health and well-being, respectively, confirm the buffering role of this process, as shown in research on Western populations. The indirect path linking physical weaknesses to well-being through temporal comparison was also stable, but weak, across countries [24]. Furthermore, results revealed a weak buffering impact of social support on well-being and self-rated health. Two additional stable effects, consistent with the literature $[13,27]$, were that financial resources increased the state of health, a favourable social comparison, the size of the social network and wellbeing, and that women reported more physical weaknesses than men, regardless of country.

\section{Differences between countries}

Sample comparisons showed a main difference in the impact of physical weakness between Chinese and other respondents. An increase in physical weaknesses was less linked to well-being, selfreported health and both dimensions of health comparison depletion for Chinese than for French, German or Russian respondents. Similarly, the positive impact of temporal comparison on self-reported health appeared in all samples except for Beijing, where physical weakness reduced the size of the social network. Besides these differences, we also found a stronger positive impact of social comparison on self-reported health for French, German and Chinese respondents than for Russian respondents.

\section{Relevance of chronological age}

Age slightly intervened in the objective and subjective evaluation of health. This observation is in line with an ageing conception in which a person's chronological age has less impact on ageing than specific types of vulnerability. As a development process characterised by asymmetry in losses and gains, in particular in health, ageing appears to be largely shaped by personal and social resources in all countries. Incomes and other resources such as education or positive social relationships that depend on social context norms had more influence than age in our study $[1,25]$.

This ageing perspective must, however, be tempered by considering that change hastens the decline of physical health at a certain age. The participants were less than 70 years old and reported few health changes, in particular in Beijing. Older seniors face more aversive events, particularly regarding health, and need to use coping strategies involving social support or social comparisons [27,53]. This complementary ageing approach could explain the weak positive impact of social resources on well-being and self-rated health in our study, as it seems to be weaker in midlife than in old age $[8,27]$.

\section{References}

1. Baltes PB, Baltes MM (1990) Successful aging: Perspectives from the behavioral sciences. Cambridge University Press, New York.

2. Brandtstädter J, Rothermund $\mathrm{K}$ (2002) The life-course dynamics of goal pursuit and goal adjustment: A two-process framework. Dev Rev 22: 117-150.

3. Freund AM, Baltes PB (1998) Selection, optimization, and compensation as strategies of life management: correlations with subjective indicators of successful aging. Psychol Aging 13: 531-543.

4. Salmela-Aro K (2009) Personal goals and well-being during critical life transitions: The four C's-Channelling, choice, co-agency and compensation. Adv Life Course Res 14: 63-73.

5. Heckhausen J, Schulz R (1993) Optimisation by selection and compensation: Balancing primary and secondary control in life span development. Int J Behav Dev 16: 287-303.

6. Schulz R, Heckhausen J (1996) A life span model of successful aging. Am Psychol 51: 702-714.

7. Heckhausen J, Wrosch C, Schulz R (2010) A motivational theory of lifespan development. Psychol Rev 117: 32-60.

8. Dainese SM, Allemand M, Ribeiro N, Bayram S, Martin M, Ehlert U (2011) Protective factors in midlife. how do people stay healthy? GeroPsych 24: 19-29.

9. Heckhausen J, Schulz R (1995) A life-span theory of control. Psychol Rev 102: 284-304.

10. Heckhausen J, Schulz R (1999) The primacy of primary control is a human universal: a reply to Gould's (1999) critique of the life-span theory of control. Psychol Rev 106: 605-609.

11. Gould SJ (1999) A critique of Heckhausen and Schulz's 1995 life-span theory of control from a cross-cultural perspective. Psychol Rev 106: 597-604.

12. Diener E, Fujita F (1995) Resources, personal strivings, and subjective well-being: a nomothetic and idiographic approach. J Pers Soc Psychol 68: 926-935.

13. Krause N, Baker E (1992) Financial strain, economic values, and somatic symptoms in later life. Psychol Aging 7: 4-14.

14. Krause N (1986) Social support, stress, and well-being among older adults. J Gerontol 41: 512-519.

15. Russell DW, Cutrona CE (1991) Social support, stress, and depressive symptoms among the elderly: test of a process model. Psychol Aging 6: 190-201.

16. Heidrich SM, Ryff CD (1993) Physical and mental health in later life: the self-system as mediator. Psychol Aging 8: 327-338.

17. Reblin M, Uchino BN (2008) Social and emotional support and its implication for health. Curr Opin Psychiatry 21: 201-205.

18. Thoits PA (2011) Mechanisms linking social ties and support to physical and mental health. J Health Soc Behav 52: 145-161.

19. Spini D, Clémence A, Ghisletta P (2007) How are temporal and social comparisons related to appraisals of self-rated health during very old age? Swiss J Psychol 66: 79-89.

20. 20. Taylor SE (1983) Adjustment to threatening events. A theory of cognitive adaptation. Am Psychol 38: 1161-1173.

21. Taylor SE, Brown JD (1988) Illusion and well-being: a social psychological perspective on mental health. Psychol Bull 103: 193-210.

22. Taylor SE, Lerner JS, Sherman DK, Sage RM, McDowell NK (2003) Portrait of the self-enhancer: well adjusted and well liked or maladjusted and friendless? J Pers Soc Psychol 84: 165-176.

23. Krause N, Shaw BA (2000) Role-specific feelings of control and mortality. Psychol Aging 15: 617-626. 
Citation: Ingrid Gilles, Alain Clemence, Nelly Courvoisier and Sylvie Sanchez (2015) Social Support, Social and Temporal Comparisons Protect Well-Being and Health between 45 and 70 Years Old in Four Urban Communities. Aging Sci 3: 133. doi:10.4172/2329-8847.1000133

Page 9 of 9

24. Robinson-Whelen S, Kiecolt-Glaser J (1997) The importance of social versus temporal comparison appraisals among older adults. J Appl Soc Psychol 27: 959-966.

25. Heckhausen J, Krueger J (1993) Developmental expectations for the self and most other people: Age grading in three functions of social comparison. Dev Psychol 29: 539-548.

26. Suls J, Marco CA, Tobin S (1991) The role of temporal comparison, social comparison, and direct appraisal in the elderly's self-evaluation of health. J Appl Soc Psychol 21: 1125-1144.

27. Li LW, Liang J (2007) Social exchanges and subjective well-being among older Chinese: does age make a difference? Psychol Aging 22: 386-391.

28. Inglehart R, Norris P (2003) Rising tide: gender equality and cultural change around the world. Cambridge University Press, New York.

29. Leung K, Bond MH (2004) Social axioms: A model for social beliefs in multi-cultural perspective. Adv Exp Soc Psychol 36: 119-197.

30. Löckenhoff CE, De Fruyt F, Terracciano A, McCrae RR, De Bolle M, et al. (2009) Perceptions of aging across 26 cultures and their culture-level associates. Psychol Aging 24: 941-954.

31. Kortchagina I, Ovtcharova L, Prokofieva L, Festy P, Verger D (2005) Conditions de vie et pauvreté en Russie, [Life conditions and poverty in Russia]. Economie et Statistique 383: 219-244.

32. World Health Organization. 2011. http://gamapserver.who.int/gho/ interactive_charts/mbd/life_expectancy/atlas.html.

33. United Nations 2009. Population, Ageing and Development. http:// social.un.org/index/LinkClick.aspx?fileticket=nq31yjeJiPc $\% 3$ dandtabid=502.

34. Phillips DR, Siu OL, Yeh AG, Cheng KH (2008) Informal social support and older persons' psychological well-being in Hong Kong. J Cross Cult Gerontol 23: 39-55.

35. Liang J (2011) Components of a meaningful retirement life--a phenomenological study of the 1950s birth cohort in urban China. J Cross Cult Gerontol 26: 279-298.

36. Nazarova I (2000) Self-rated health and occupational conditions in Russia. Soc Sci Med 51: 1375-1385.

37. Cheng ST, Fung H, Chan A (2007) Maintaining self-rated health through social comparison in old age. J Gerontol B Psychol Sci Soc Sci 62: P277-285.

38. Kunzmann U, Little TD, Smith J (2000) Is age-related stability of subjective well-being a paradox? Cross-sectional and longitudinal evidence from the Berlin Aging Study. Psychol Aging 15: 511-526.
39. Sales AE, Estabrooks CA, Valente TW (2010) The impact of social networks on knowledge transfer in long-term care facilities: Protocol for a study. Implement Sci 5: 49.

40. Lalive d'Epinay C, Spini D (2008) Les Années fragiles. La Aie après 80 Ans, [Frail years. Life beyond 80 Years]. Presses de l'Université de Laval, Laval.

41. European health interview survey EHIS - collection round 2008. http:// epp.eurostat.ec.europa.eu/cache/ITY_SDDS/en/hlth_ehis_esms.htm

42. Wang R, Treul S, Alverno L (1975) A brief self-assessing depression scale. J Clin Pharmacol 15: 163-167.

43. Clémence A, Karmaniola A, Green EGT, Spini D (2007) Disturbing life events and wellbeing after 80 years of age: a longitudinal comparison of survivors and the deceased over five years. Ageing and Soc 27: 195-213.

44. Byrne BM (2004) Testing for multigroup invariance using AMOS graphics: A road less traveled. Struct Equ Modeling 11: 272-300.

45. Stephenson MT, Holbert RL (2003) A Monte Carlo simulation of observable versus latent variable structural equation modeling techniques. Communic Res 30: 332-354.

46. Byrne BM (1991) Burnout: Investigating the impact of background variables for elementary, intermediate, secondary, and university educators. Teach Teach Educ 7: 197-209.

47. Kline RB (1998) Principles and practice of structural equation modeling. Guilford Press, New York.

48. Hu L, Bentler P (1999 Cutoff criteria for fit indices in covariance structure analysis: Conventional criteria versus new alternatives. Struct Equ Modeling 6: 1-55.

49. Zhang Y, Goza FW (2006) Who will care for the elderly in China? A review of the problems caused by China's one-child policy and their potential solutions. J Aging Stud 20: 151-164.

50. Yi Z, Deqing W (2000. A regional analysis of divorce in China since 1980. Demography 37: 215-219.

51. Cooney RS, Li J (1994) Household registration type and compliance with the "one child" policy in China, 1979-1988. Demography 31: 21-32.

52. Krause N (2005) Exploring age differences in the stress-buffering function of social support. Psychol Aging 20: 714-717. 